\title{
Rapidly Deteriorating Kidney Function in a Young Man Previously Diagnosed with Membranous Nephropathy
}

\author{
Giovani Gadonski ${ }^{\mathrm{a}}$ Carlos E. Poli-de-Figueiredo ${ }^{\mathrm{a}}$ Fernando C. Fervenza ${ }^{\mathrm{b}} \quad$ Ajay K. Singh ${ }^{\mathrm{c}}$ \\ Fernando M. Tettamanzy ${ }^{a}$ Ivan C.F. Antonello ${ }^{a}$ Domingos O. d'Avila ${ }^{a}$ \\ Coordinators: Fernando C. Fervenzab Ajay K. Singh ${ }^{c}$ \\ ${ }^{a}$ Hospital São Lucas, Instituto de Pesquisas Biomédicas/Faculdade de Medicina/PUCRS, Porto Alegre, Brazil; \\ ${ }^{b}$ Division of Nephrology and Hypertension, Mayo Clinic College of Medicine, Rochester, Minn., USA and \\ 'Renal Division, Brigham and Women's Hospital, Harvard Medical School, Boston, Mass., USA
}

\section{Debater: Giovani Gadonski}

Division of Nephrology, Programa de Pós-Graduação em Medicina e Ciências da Saúde (Nefrologia)

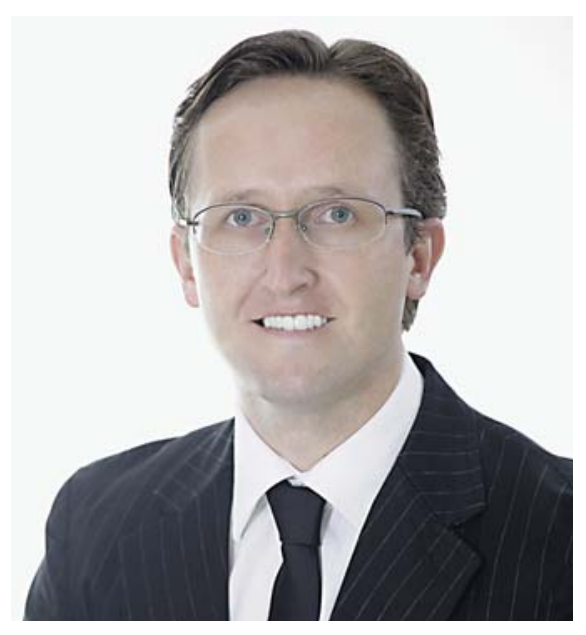

Giovani Gadonski, 34 years old, graduated (1999) in Medicine from Fundação Faculdade Federal de Ciências Médicas, Porto Alegre, Brazil. He went on to complete his Residence in Medicine (2000-2001) and Nephrology (2002-2003) at Hospital São Lucas, Porto Alegre, Brazil. He then entered a postgraduate program in Medicine and Health Sciences at the Pontifical Catholic University of
Rio Grande do Sul which he completed in 2006, following a 1-year training period at the Physiology Department, University of Mississippi, USA. He is currently a nephrologist and researcher at São Lucas Hospital and the Nephrology Laboratory, Institute of Biomedical Research, Pontifical Catholic University of Rio Grande do Sul, Porto Alegre, Brazil.

\section{Case Presentation \\ Carlos E. Poli-de-Figueiredo o ${ }^{a}$ Fernando M. Tettamanzy ${ }^{b}$ \\ ${ }^{a}$ Faculdade de Medicina, Instituto de Pesquisas Biomédicas/PUCRS, Porto Alegre, and ${ }^{\text {bHospital São }}$ Lucas, Instituto de Pesquisas Biomédicas/PUCRS, Porto Alegre, Brazil}

Dr. Poli-de-Figueiredo: An 18-year-old black male came to the Emergency Room of Hospital São Lucas/ PUCRS - a privately owned University Hospital linked to the Unified Health System, a federal government-covered program providing free healthcare to all Brazilian citizens - complaining of nausea and vomiting for the last 2 days. One month earlier he had been diagnosed with acute respiratory infection. At that time, serum creatinine was $88.4 \mu \mathrm{mol} / \mathrm{l}$; urinalysis showed protein $3+$, hemoglobin 3+, and 10 leukocytes and $20 \mathrm{RBC} / \mathrm{hpf}$. The urine protein/creatinine ratio (UPCR) was then 7.3. WBC

\section{KARGER}

두 2010 S. Karger AG, Basel

Fax +41613061234 E-Mail karger@karger.ch www.karger.com 
count was $11,400 / \mu 1$ with 1,140 eosinophils. Stool analysis demonstrated Giardia lamblia and he was treated with albendazole/thiabendazole). The patient was discharged on amoxicillin-clavulanate as treatment for the acute respiratory infection. Serum creatinine was now 583

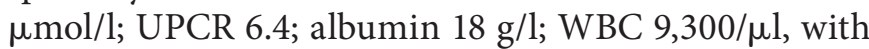
747 eosinophils/ $\mu$ l. Urinalysis showed protein $3+$, hemoglobin 3+, 30 leukocytes and 35 RBC/hpf. Antinuclear antibodies (ANA), anti-neutrophil cytoplasmic antibodies (ANCA) and anti-glomerular basement membrane (GBM) antibodies were negative; $\mathrm{C} 3$ was 0.85 (normal range 0.88-1.65) g/l and C4 0.29 (0.14-0.44) g/l; HBsAg, anti-HCV and anti-HIV were negative. Doppler ultrasound of the renal arteries and veins was normal. Nephrotic syndrome evolved and renal function declined, and despite treatment with 3 intravenous pulses of methylprednisolone (500 $\mathrm{mg}$ each), the patient required hemodialysis.

The patient had a history of nephrotic syndrome which presented 2 years earlier. At that time, UPCR was 8.8; Creactive protein was 12,900 (normal $<10,000) \mu \mathrm{g} / \mathrm{l}$; ANA and anti-DNA antibodies were negative; C3 was 1.34 $(0.90-1.80) \mathrm{g} / \mathrm{l}$ and $\mathrm{C} 40.34(0.10-0.40) \mathrm{g} / \mathrm{l}$; antistreptolysin-O antibody was $<49,000 \mathrm{Ul} / \mathrm{l} ; \mathrm{HBsAg}$, anti-HCV and anti-HIV antibodies were negative. Chest radiograph revealed bilateral pleural effusions. Renal ultrasound showed a right kidney of $12.3 \mathrm{~cm}$ and left kidney of 12.8 $\mathrm{cm}$, with no obstruction. A percutaneous kidney biopsy was then performed and the diagnosis was membranous nephropathy.

Dr. Tettamanzy: The first renal biopsy specimen obtained 2 years previously contained 10 apparently normal glomeruli. On silver stain there was mild diffuse thickening of glomerular basement membrane with focal spike formation (fig. 2). Immunofluorescence staining on frozen sections showed global, coarse granules, along the peripheral capillary loops that were positive for immunoglobulin G (IgG) and C3, and segmental deposits of C1q. There was no staining with antibodies against IgA, IgM, fibrinogen, $\kappa$ and $\lambda$ chains. No electron microscopy was performed. Pathological findings were interpreted as compatible with membranous nephropathy. A recent review of almost 10,000 native kidney biopsies of Brazilian patients showed membranous nephropathy to be the second (20.7\%) most frequently found histological lesion [1].

Dr. Poli-de-Figueiredo: Based on the clinical presentation and the renal pathology a diagnosis of idiopathic membranous nephropathy was made. Treatment was initiated with enalapril (5 mg/day), simvastatin ( $20 \mathrm{mg} /$ day) and furosemide $(80 \mathrm{mg} /$ day). Additionally, he was given
3 intravenous methylprednisolone (500 $\mathrm{mg} /$ day) pulses, starting on day 4. At discharge, his condition was improved - his weight was $58.5 \mathrm{~kg}$ (a total loss of $13.5 \mathrm{~kg}$ ) and he no longer had detectable edema. Laboratory data showed $1+$ protein on dipstick, $<5 \mathrm{RBC} / \mathrm{hpf}$ on microscopy. Blood urea nitrogen was $8.9 \mathrm{mmol} / \mathrm{l}$, creatinine 91.5 $\mu \mathrm{mol} / \mathrm{l}$, albumin $151.5 \mu \mathrm{mol} / \mathrm{l}$, and UPCR 7.5. At discharge he was taking prednisone ( $1 \mathrm{mg} / \mathrm{kg} /$ day), simvastatin, enalapril, furosemide, aspirin, sulfisoxazole-trimethroprim and albendazole (as G. lamblia was detected on stool examination).

At 1 year of follow-up, serum creatinine was $80 \mu \mathrm{mol} / \mathrm{l}$ and UPCR 2.4. Prednisone dose had been tapered down to $7.5 \mathrm{mg} /$ day. Creatinine was $88.4 \mu \mathrm{mol} / \mathrm{l}$; UPCR 0.2 ; normal RBC count, with 10,800 WBC and 2,419 eosinophils. Stool examination was negative.

\section{Case Discussion}

Giovani Gadonski' , Ajay K. Singh ${ }^{b}$, Domingos O. d'Avilac, Ivan C.F. Antonello ${ }^{C}$, Fernando Fervenza ${ }^{d}$

${ }^{a}$ Nephrology Division, Instituto de Pesquisas

Biomédicas/PUCRS, Porto Alegre, Brazil;

bHarvard Medical School, Brigham and Women's

Hospital, Boston, Mass., USA; 'Faculdade de Medicina/

Instituto de Pesquisas Biomédicas/PUCRS, Porto Alegre,

Brazil, and dMayo Clinic College of Medicine, Rochester,

Minn., USA

Dr. Gadonski: In a patient with preexisting membranous nephropathy with a favorable response to initial treatment, followed by rapidly falling renal function requiring dialysis, some common conditions associated with renal failure must be investigated, such as acute kidney injury (AKI). This is a rare, spontaneous, complication of nephrotic syndrome which can be associated with hypovolemia due to excessive diuresis, acute interstitial nephritis, sepsis or renal vein thrombosis $[2,3]$. Acute interstitial nephritis is a common situation associated with the use of antibiotic or non-steroidal anti-inflammatory drugs [4]. It was known from the medical history that he had taken antibiotics 1 month prior to the current hospitalization. However, there is no description of a concomitant skin rash. No investigation for urinary eosinophils was undertaken, yet the presence of a high number of blood eosinophils falling after treatment for intestinal giardiasis makes the diagnosis less likely. The possibility of a delayed abnormal response to antibiotic exposure cannot be totally ruled out. Another possibility would be 
Fig. 1. Differential diagnosis of AKI in a patient with known membranous nephropathy.

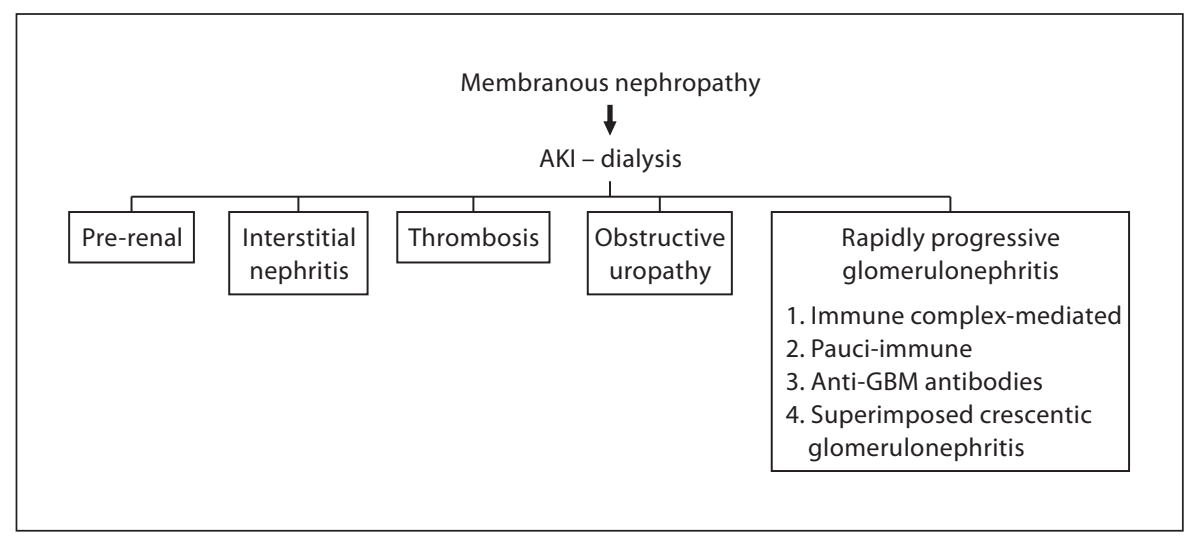

renal vein thrombosis which may complicate the nephrotic syndrome, and result in severe renal failure. Such event was described as early as 1977 [5]. One third of the reported series - either membranous or membranoproliferative nephropathy - had renal vein thrombosis. More recent surveys have demonstrated a similar prevalence (from 10 to 30\%). Urinary loss of antithrombin III, protein $\mathrm{S}$ and $\mathrm{C}$ have been associated with the occurrence of renal vein thrombosis [6]. Doppler ultrasonography or renal venography are useful tools in the diagnosis but were negative in the current case. Similarly, an obstructive uropathy was ruled out by a normal urinary tract ultrasound.

I now want to present and discuss figure 1 in order to address the differential diagnosis of AKI in a patient with known membranous nephropathy.

Once the most common conditions associated with AKI in a patient with membranous nephropathy have been ruled out, we must consider less common causes, such as rapidly progressive glomerulonephritis which can be revised according to the mechanisms involved.

Immune complex-mediated (systemic lupus erythematosus, SLE) nephropathy develops in approximately $25 \%$ of patients; it is more frequently seen in black people and women. Lupus nephritis is largely mediated by glomerular immune complex deposition [6]. The immunofluorescence slides, in the current case, demonstrated GBM IgG, C3 and C1q deposition, even though C1q deposits were quite weak. However, no further clinical or laboratorial criteria for SLE, such as ANA, were ever demonstrated. Yet one should not fully rule out SLE as the kidney lesions may go from one histological pattern into another - it is not uncommon for a class III lesion to evolve to class IV lupus nephritis. Both classes III and IV can transform into membranous (class V) nephropathy, either spontaneously or under therapy. Even less common, yet possible, is a transformation from a membranous to a proliferative lesion [8]. However, the current patient had negative ANA and anti-DNA, with normal complements, and a previous renal biopsy with membranous change had no proliferation and he did not have the full house that one is used to seeing on immunofluorescence, which renders the hypothesis less likely.

The fact that the patient had a history of respiratory infection 1 month prior to the last hospitalization raises the possibility of a post-infectious glomerulonephritis with accompanying nephrotic proteinuria and progressively declining renal function [9]. The fact that serum antistreptolysin-O levels and complement levels were normal make this hypothesis unlikely.

IgA nephropathy is another possibility for an immune complex-mediated glomerulonephritis. IgA nephropathy is the most frequent primary glomerulonephritis, affecting individuals of all ages. Cases presenting with IgA subepithelial deposits have been reported in Japan [10]. Yet in the first biopsy no IgA deposits were found.

Focal segmental glomerulosclerosis (FSGS) should also to be considered. FSGS is the most common histological pattern in idiopathic nephrotic syndrome among black individuals [3]. FSGS, especially the collapsing variant, may progress with loss of renal function and heavy proteinuria. It is most frequently associated with HIV [11], which was negative in this patient. However, reports of collapsing glomerulonephritis, coexisting with membranous nephropathy have been published in HIV-negative patients [12].

Another possibility would be systemic vasculitis. In a series of 10 patients, vasculitis transformation of a membranous nephropathy, with rapid deterioration of renal function requiring dialysis, was demonstrated [13]. Vas- 
culitis, or crescentic glomerulonephritis, is rarely seen in membranous nephropathy, except in cases associated with SLE. However, some reported cases could not be linked to SLE (ANA-negative, normal complement). Among 9 patients, 4 were ANCA-positive and 6, tested for anti-GBM, were negative. Vasculitis should be thought of when there is evidence of capillary inflammation, hematuria and proteinuria. The patient under discussion had hematuria, proteinuria and rapidly falling kidney function, yet no systemic evidence of small vessel inflammation.

Pauci-immune crescentic glomerulonephritis is an entity strongly associated with the presence of circulating ANCA. Its clinical and pathological variants are ChurgStrauss syndrome, Wegener granulomatosis and microscopic polyangiitis. The majority of patients with systemic vasculitis have constitutional features of inflammatory disease - fever, arthralgia, myalgias and weight loss. This patient had eosinophilia, yet no history of asthma. However, G. lamblia was isolated from stools in February 2007, which might justify the WBC abnormality. ChurgStrauss vasculitis has the lowest frequency of renal involvement [14], making this diagnosis unlikely. Patients with Wegener's granulomatosis have necrotizing inflammatory lesions associated with mostly respiratory tract granulomas. Sinus inflammation and bleeding, as well as definite X-ray or tomography images usually are apparent [15]. No such findings were evidenced in this patient. Some individuals may present with vasculitis and no granulomatous inflammation, asthma or eosinophilia as in microscopic polyangiitis [16]. Approximately $50 \%$ of the patients with Churg-Strauss syndrome are ANCAnegative, but when presenting with kidney involvement are usually positive for MPO-ANCA (ANCA-negative glomerulonephritis associated with non-asthmatic Churg-Strauss syndrome) [17]. Also, Immune complexmediated vasculitis may present with glomerular epithelial proliferation and rapidly progressive renal failure in association with cryoglobulins and C-virus hepatitis or membranoproliferative nephropathy [18]. This patient was C-virus-negative and had normal complements.

Vasculitis mediated by anti-GBM antibodies is a nother possibility. Cases evolving as rapidly progressive glomerulonephritis (going from membranous nephropathy to anti-GBM glomerulonephritis) have been reported [19]. Anti-GBM disease is more prevalent among white individuals and is characterized by pulmonary hemorrhage, yet one third of the patients may present with isolated nephritis, with hematuria, proteinuria and rapidly progressive renal failure [20]. Negative anti-GBM antibodies make the diagnosis unlikely. However, a few patients have antibodies secondary to IgA anti-GBM antibodies [21], which are not tested on routine anti-GBM testing.

Last but not least, I will consider crescentic glomerulonephritis, as this pathological presentation is clinically characterized by rapidly decreasing renal function. In an extensive Brazilian kidney biopsy study, it occurred in only $1.7 \%$ of the evaluated tissue samples [1]. Yet patients presenting with AKI and anasarca, dependent upon a crescentic glomerulonephritis superimposed on a previously diagnosed membranous nephropathy, have been reported previously $[22,23]$. This is a rare condition, but must be considered, since the patient has nephrotic range proteinuria and no extra-renal manifestations, as previously discussed. Additionally, there are other reports associating membranous nephropathy with crescentic glomerulonephritis, or progression of membranous nephropathy to acute crescentic, rapidly progressive glomerulonephritis [24].

In summary, this may be a rare presentation of membranous nephropathy. From my point of view, it is more likely that this patient has ANCA-negative, anti-GBM or a de novo crescentic glomerulonephritis superimposed on a primary membranous nephropathy.

Dr. Singh: Thank you for your outstanding case discussion. I also thought about the differential diagnosis, and I wonder whether you think this patient might have a chronic infection from G. lamblia. He had previously been treated with albendazole, and in 2007 G. lamblia was detected in his stools. This would not explain the renal failure but could this be a case of secondary membranous nephropathy associated with G. lamblia?

Dr. Gadonski: Yes, that is a possibility. He had a quite high eosinophil count, suggesting that a particularly intense immunological reaction was taking place. Giardiasis may, or may not, evolve with eosinophilia and other parasitic infections, such as strongyloidiasis, should not be discarded [25]. However, association between G. lamblia infection and nephrotic syndrome has recently been suggested [26]. Yet it has not been linked to a rapidly progressive course as this patient lately had. Incidentally, more than $20 \%$ of preschool-aged children in a survey of a poor local neighborhood were found infected by G. lamblia, suggesting a high prevalence of the parasite among deprived inhabitants in Porto Alegre [27].

Dr. Singh: Because FSGS is a focal lesion, only 10 glomeruli may be insufficient to rule out a focal disease. Since this was one of the hypotheses raised in your differential diagnosis, do you think it is still possible to consider this diagnosis? 


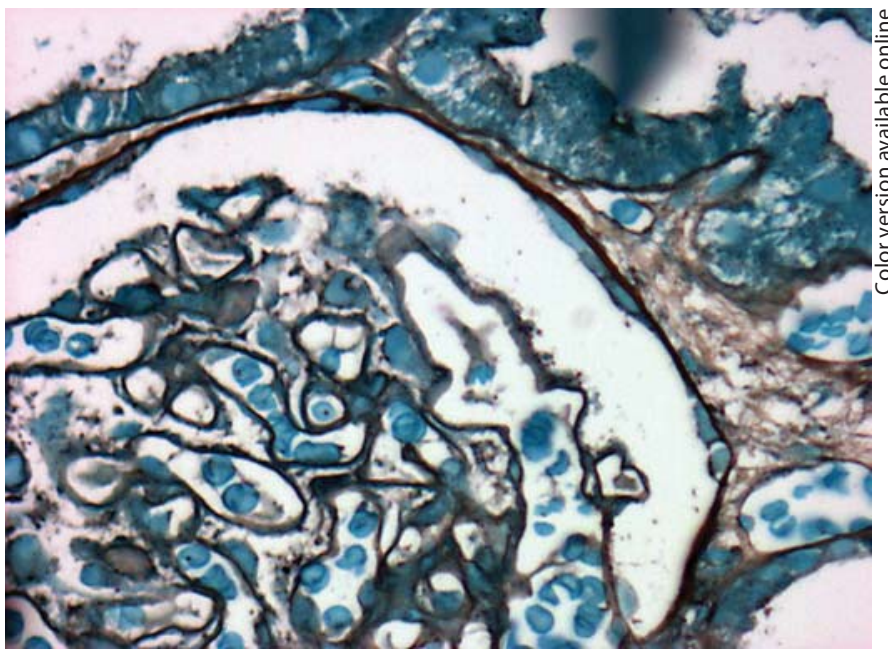

Fig. 2. First biopsy: spikes can be seen on silver stain. $\times 400$.

Dr. Gadonski: I agree with your observation - one glomerulus with focal sclerosis would settle the diagnosis. Repeating the renal biopsy would eventually be necessary.

Dr. d'Avila: The first biopsy showed IgG and C3 deposits in all glomeruli, making membranous nephropathy a more likely diagnosis.

Dr. Singh: I am considering this possibility as a study showed coexistence of FSGS with a membranous glomerulopathy [28]. Regarding the second presentation of renal failure, I wonder about the possibility of acute interstitial nephritis, since he took amoxicillin and clavulanate, and had 35 leukocytes in urine. Also, in severe nephrotic syndrome, acute tubular necrosis can be considered.

Dr. Gadonski: Against that possibility, there is no history of clinically relevant hypovolemia, described as hypotension or other relevant clues.

Dr. Singh: Years ago I was presented a case with severe nephrotic syndrome and I reviewed it for a CPC $[29,30]$. One of the most common causes of renal failure in this setting is acute tubular necrosis, which is usually underestimated. This is even more common in patients over 60 years of age.

Dr. Gadonski: Dr. Antonello has been following this patient since his first hospitalization as well as after discharged. Could you please add further information?

Dr. Antonello: When this patient was first biopsied, we had decided not to use cyclophosphamide because he had pneumonia. Upon improving, we discharged him from hospital to be followed at the outpatient clinic. Unfortu-

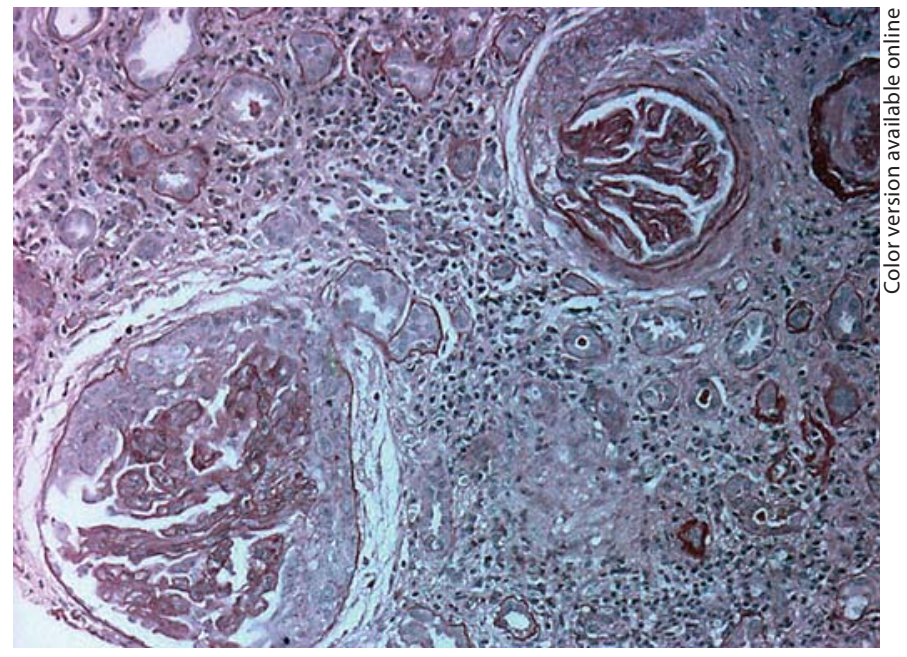

Fig. 3. Epithelial crescents are readily apparent on the second biopsy. PAS. $\times 100$.

nately, he was lost to follow-up and kept using $20 \mathrm{mg}$ of prednisone for 2-3 months. Prednisone was tapered down to $5 \mathrm{mg}$ /day and urinary protein remained low, which made us keep it at low dose.

Dr. Singh: One additional possibility to be considered is HIV. Laurinavicius et al. [31] published a very nice series in Kidney International in which they reported cases with HIV nephropathy and negative serology. That is the reason why I think you should keep the possibility of HIV.

Dr. Fervenza: This patient is nephrotic and has an active sediment. Clinically he behaves as though he has rapidly progressive glomerulonephritis. All common causes have been ruled out as per our discussant. If I had to bet, I would say that he has crescentic glomerulonephritis or acute interstitial nephritis.

Dr. Gadonski: Or both.

Dr. Singh: I would bet that he has acute interstitial nephritis and acute tubular necrosis, but not crescentic glomerulonephritis.

\section{Final Pathological Diagnosis}

Dr. Tettamanzy: The second renal biopsy specimen contained 10 glomeruli. On light microscopy, crescents were quite evident in almost all the glomeruli (fig. 3). On periodic acid-Schiff (PAS) and silver staining, there was a diffuse and uniform thickening of GBM with extensive spike formation (fig. 4) and also a global and diffuse ex- 


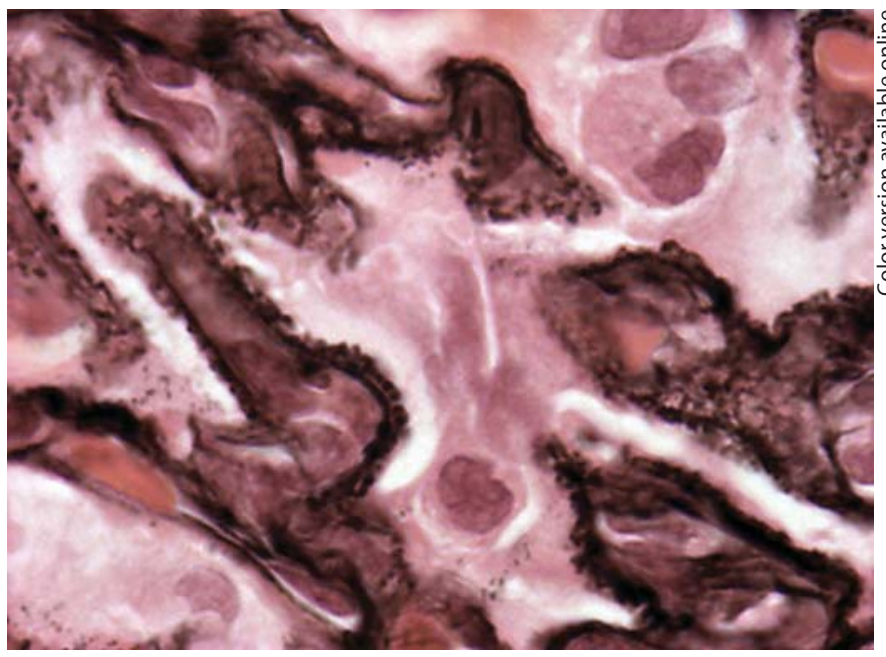

Fig. 4. Second biopsy showing spikes along the basement membrane. Silver stain. $\times 400$.

tracapillary cellular proliferation (crescent formation) without fibrinoid necrosis or intraglomerular thrombosis (fig. 3, 5). There was one globally sclerotic glomerulus and severe interstitial mononuclear cell infiltration and edema. No vascular lesions were found. Immunofluorescence staining on frozen sections showed the same deposits as those found on the first biopsy. Pathological findings were interpreted as membranous nephropathy with extracapillary proliferation.

Dr. Fervenza: Thank you very much Dr. Tettamanzy. Before we end this CPC, are there any questions or comments that anyone would like to ask or make?

Dr. d'Avila: What would be your recommendations for treatment of this patient?

Dr. Fervenza: The treatment of crescentic glomerulonephritis superimposed on a patient with idiopathic

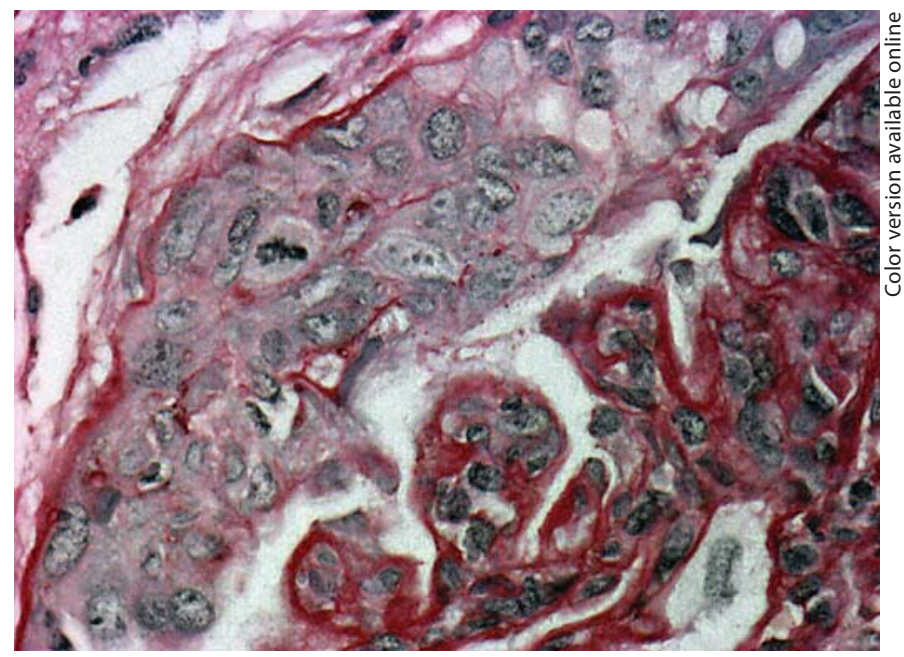

Fig. 5. Higher magnification view of crescents filling the capsular space. PAS. $\times 400$.

membranous nephropathy is said to be equal to that of patient with a crescentic glomerulonephritis secondary to ANCA-associated vasculitis. Traditionally, this consists of high-dose corticosteroids in combination with cyclophosphamide. However, these patients are rare and no specific studies or outcome measures are available regarding this patient population. It is also unknown if these patients can respond to other forms of immunosuppressive therapy such as corticosteroids combined with mycophenolate mofetil or with rituximab. As opposed to patients with ANCA-associated vasculitis, there are no data to support or refute the use of plasmapheresis in patients who present with advanced renal failure.

We must now end, and thank you all for participating in this interesting and very useful CPC.

\section{References}

1 Polito MG, de Moura LA, Kirsztajn GM: An overview on frequency of renal biopsy diagnosis in Brazil: clinical and pathological patterns based on 9,617 native kidney biopsies. Nephrol Dial Transplant 2010;25:490-496.

$\checkmark 2$ Koomans HA: Pathophysiology of acute renal failure in idiopathic nephrotic syndrome. Nephrol Dial Transplant 2001; 16:c221-c224.

>3 Hulll RP, Goldsmith JA: Nephrotic syndrome in adults. BMJ 2008;336:c1185-c1189.
4 John R, Herzenberg AM: Renal toxicity of therapeutic drugs. J Clin Pathol 2009; 62:c505-c515.

5 Kutcher R, Cohen JR, Gordon DH: Glomerulonephritis and nephrotic syndrome complicated by renal vein thrombosis and pulmonary emboli: report of two cases. Am J Roentgenol 1977;128:c447-c449.

-6 Nickolas TL, Radhakrishnan J, Appel GB: Hyperlipidemia and thrombotic complications in patients with membranous nephropathy. Semin Nephrol 2003;23:c406-c411.
Austin HA, Illei GG: Membranous lupus nephritis. Lupus 2005;14:c65-c71.

-8 Baldwin DS, Gluck MC, Loweinstein J, Gallo GR: Lupus nephritis. Clinical course as related to morphologic forms and their transitions. Am J Med 1977;62:c12-c30.

$\checkmark 9$ Kanjanabuch T, Kittikowit W, Eiam-Ong S: An update on postinfectious glomerulonephritis worldwide. Nat Rev Nephrol 2009; 5:c259-c269. 
-10 Doi T, Kanatsu K, Nagai H, Kohrogi N, Hamashima Y: An overlapping syndrome of IgA nephropathy and membranous nephropathy? Nephron 1983;35:c24-c30.

11 Wakai S, Magil A: Focal glomerulosclerosis in idiopathic membranous glomerulonephritis. Kidney Int 1992;41:c428-c434.

$\checkmark 12$ Al-Shamari A, Yeung K, Levin A, Taylor P, Magil A: Collapsing glomerulopathy coexisting with membranous glomerulonephritis in native kidney biopsies: a report of $3 \mathrm{HIV}$ negative patients. Am J Kidney Dis 2003; 42:c591-c595.

$\checkmark 13$ Tse WY, Howie AJ, Adu D, Savage COS, Richards NT, Wheeler DC, Michael J: Association of vasculitic glomerulonephritis with membranous nephropathy: a report of 10 cases. Nephrol Dial Transplant 1997; 12:c1017-c1027.

-14 Solans R, Bosch JA, Perez-Bocanegra C, Selva A, Huquet P, Alijotas J, Orriols R, Armadans L, Vilardell M: Churg-Strauss syndrome: outcome and long-term follow-up of 32 patients. Rheumatology 2001;40:c763c771.

-15 Hoffman GS, Kerr GS, Leavitt RY, Hallahan CW, Lebovics RS, Travis WD, Rottem M, Fauci AS: Wegener's granulomatosis: an analysis of 158 patients. Ann Intern Med 1992;116:c488-c498.

16 Jennette JC, Thomas DB, Falk RJ: Microscopic polyangiitis (microscopic polyarteritis). Semin Diagn Pathol 2001;18:3-13.

$\checkmark 17$ Chemmalakuzhy AJ, Zhou XJ, Hedayati SS: ANCA-negative glomerulonephritis associated with nonasthmatic Churg-Strauss syndrome. Nat Clin Pract Nephrol 2008;4:c568c574.
18 Rossi P, Bertani T, Baio P: Hepatitis C virusrelated cryoglobulinemic glomerulonephritis: long-term remission after antiviral therapy. Kidney Int 2003;63:c2236-c2241.

19 Klassen J, Elwood C, Grossberg AL, Milgrom F, Montes M, Sepulveda M, Andres GA: Evolution of membranous nephropathy into anti-glomerular-basement-membrane glomerulonephritis. N Engl J Med 1974; 290:c1340-c1344.

20 Troxell ML, Saxena AB, Kambham N: Concurrent anti-glomerular basement membrane disease and membranous glomerulonephritis: a case report and literature review. Clin Nephrol 2006;66:c120-c127.

21 Ho J, Gibson IW, Zacharias J, Fervenza F, Colon S, Borza DB: Antigenic heterogeneity of IgA anti-GBM disease: new renal targets of IgA autoantibodies. Am J Kidney Dis 2008;52:c761-c765.

22 James SH, Lien YH, Ruffenach SJ, Wilcox GE: Acute renal failure in membranous glomerulonephropathy: a result of superimposed crescentic glomerulonephritis. J Am Soc Nephrol 1995;6:c1541-c1546.

23 Hall AM, Symington EM, Sampson AS, Kwan JTC: Crescentic transformation of membranous glomerulopathy: a reversible condition. Nephrol Dial Transplant 2006; 21:c1136-c1137.

24 Charney DA, Singh A, Aaron N: A male nephrotic patient with rapid decline in renal function. Nephrol Dial Transplant 2005; 20:c1263-c1266
25 Wong TY, Szeto CC, Lai FF, Mak CK, Li PK: Nephrotic syndrome in strongyloidiasis: remission after eradication with anthelmintic agents. Nephron 1998;79:c333-c336.

26 Copelovitch L, Ol OS, Taraquinio S, Chanpheaktra N: Childhood nephrotic syndrome in Cambodia: an association with gastrointestinal parasites. J Pediatr 2010;156:76-81.

27 Morrone FB, Carneiro JA, dos Reis C, Cardozo CM, Ubal C, De Carli G: Study of enteroparasites infection frequency and chemotherapeutic agents used in pediatric patients in a community living in Porto Alegre, RS, Brazil. Rev Inst Med trop Sao Paulo 2004;46:77-80.

28 Matsuo T, Mori Y, Hashimoto T, Tajima M, Okado T, Tsukamoto Y, Kobayashi Y, Sasaki $\mathrm{S}$ : Coexistence of idiopathic membranous glomerulonephritis and idiopathic focal segmental glomerulosclerosis. Clin Nephrol 2005;64:c490-c492.

29 Williams WW Jr, Ecker JL, Thadhani RI, Rahemtullah A: Case records of the Massachusetts General Hospital. Case 38-2005. A 29-year-old pregnant woman with the nephrotic syndrome and hypertension. N Engl J Med 2005;353:2590-2600.

30 Case records of the Massachusetts General Hospital. Weekly clinicopathological exercises. Case 3-2000. A 66-year-old woman with diabetes, coronary disease, orthostatic hypotension and the nephrotic syndrome. $\mathrm{N}$ Engl J Med 2000;342:c264-c273.

-31 Laurinavicius A, Hurwitz S, Rennke HG: Collapsing glomerulopathy in HIV and nonHIV patients: a clinicopathological and follow-up study. Kidney Int 1999;56:c2203c2213. 